FORMATION Formation emploi

Revue française de sciences sociales

133 | Janvier-Mars 2016

Heurs et malheurs de l'apprentissage en Suisse

\title{
Le Certificat fédéral de capacité : la fin d'une centralité ?
}

Federal VET Diploma : end of a key-position?

Fähigkeitszeugnis : Ende eines bevorzugten Berufsabschlusses?

El Certificado federal de capacidad : ¿el fin de una centralidad?

Nadia Lamamra et Gilles Moreau

\section{CpenEdition}

Journals

Édition électronique

URL : http://journals.openedition.org/formationemploi/4661

DOI : 10.4000/formationemploi.4661

ISSN : 2107-0946

Éditeur

La Documentation française

Édition imprimée

Date de publication : 20 avril 2016

Pagination : 101-120

ISSN : 0759-6340

Référence électronique

Nadia Lamamra et Gilles Moreau, «Le Certificat fédéral de capacité : la fin d'une centralité ? »,

Formation emploi [En ligne], 133 | Janvier-Mars 2016, mis en ligne le 21 avril 2018, consulté le 30 octobre 2020. URL : http://journals.openedition.org/formationemploi/4661 ; DOI : https://doi.org/ 10.4000/formationemploi.4661

(c) Tous droits réservés 


\section{Le Certificat fédéral de capacité : la fin d'une centralité?}

NADIA LAMAMRA
Sociologue, Institut fédéral des hautes études en formation professionnelle, EHB IFFPIUFFP,

Lausanne

GILLES MOREAU Sociologue, Groupe de recherches et d'études sociologiques du Centre Ouest, GRESCO, université de Poitiers

Résumé

Le Certificat fédéral de capacité : la fin d'une centralité ?

Le CFC, Certificat fédéral de capacité, est un diplôme historique en Suisse. Depuis les années 1930, il est la référence pour la formation professionnelle de niveau secondaire. Grâce à lui, la formation par apprentissage s'est imposée en Suisse. Mais alors que son image reste forte, les réformes entreprises depuis les années 1990 signent la fin de sa prééminence dans le système suisse des diplômes. De quasi monopolistique, il est devenu minoritaire, y compris dans la voie professionnelle ; cela est dû à l'apparition de nouveaux diplômes et à l'élévation constante des niveaux de formation, notamment dans le tertiaire (supérieur).

Mots clés : enseignement technique-professionnel, apprentissage, certification, politique de l'éducation, statistique de l'éducation, Suisse

Abstract

Federal VET Diploma: end of a key-position ?

The Federal VET Diploma is a historical qualification in Switzerland. Created in the 1930's, it has become a reference diploma at upper-secondary level. At the same time, the dual-track has become the predominant form of vocational education and training in Switzerland. Nevertheless, while the Federal VET Diploma has retained its very solid reputation, the reforms undertaken in the 1990's put an end to its predominant status within the Swiss system of diplomas. It has moved from a virtually monopolistic situation to a minority one, due to the creation of new diplomas and the constant elevation of educational levels, in particular at tertiary education.

Keywords: technical \& vocational education, apprenticeship, certification, education policy, education statistics, Switzerland

Journal of Economic Literature: I 21, I 28

Traduction : Auteur.e 
Le Certificat fédéral de capacité (CFC) est, en Suisse, ce que fut le Certificat d'aptitude professionnelle (CAP) en France : un "diplôme-témoin " de la mise en forme scolaire des savoirs et savoir-faire de métiers, et de la formation des ouvrier.e.s et employé.e.s.

Mais contrairement au CAP, le CFC demeure, outre-Jura, le diplôme de référence pour les apprenti-e-s en alternance ou, plus rarement, pour celles et ceux en écoles dites à pleintemps ${ }^{1}$. Situé au niveau du secondaire II dans " le système suisse de diplômes» (Waardenburg, 2011), il s'adresse à des jeunes âgé-e-s de 15 ans ou plus à l'issue du secondaire obligatoire (secondaire I) et est « le diplôme de référence de l'ensemble des filières de formation post-obligatoire ", dans un pays où "les $3 / 4$ des jeunes de l'enseignement secondaire II se retrouvent à l'intérieur d'une des filières professionnelles" (Cortesi \& Imdorf, 2013, p. 91).

C'est dire sa centralité dans l'apprentissage en Suisse, mais plus généralement dans la société helvétique ${ }^{2}$ : n’a-t-on pas vu certaines très hautes fonctions occupées par des titulaires d'un CFC ? Ainsi Sergio Ermotti ${ }^{3}$, l'actuel PDG de l'Union des Banques Suisses (UBS) ou encore Ueli Maurer ${ }^{4}$, le ministre de la Défense, président de la Confédération en 2014, sont tous deux titulaires d'un CFC. A croire qu'il ouvre tous les possibles.

Un diplôme est plus qu'une simple catégorie statistique permettant de regrouper les jeunes par niveau de formation. En tant qu'attestation prédictive (Brucy, 1998), il a une fonction d'insertion sur le marché du travail, mais vaut aussi pour l'usage que fait son titulaire des savoirs qui y sont associés, ainsi que par sa dimension symbolique. Il a aussi pour caractéristique, surtout lorsqu'il est professionnel, d'avoir fait du savoir et du savoir-faire, longtemps considérés comme propriété privée (Kaplan, 2001), un «bien public " auquel tous et toutes peuvent prétendre, même si tous et toutes n'y accèdent pas (Millet, Moreau, 2011). En ce sens, il est aussi le produit de politiques publiques.

Pour autant, les diplômes ont été peu étudiés. En France, si l'on excepte La barrière et le niveau, d'Edmond Goblot (1925) et des travaux d'historiens (Brucy 1998 ; Cabanel, 2002 ; Marchand, 2010) ou de sociologues (Maillard, 2008 ; Brucy et al., 2013), les diplômes demeurent un chantier relativement déserté. Certes, quelques publications ont, dans la seconde moitié du XXe siècle, marqué la problématique des diplômes, à l'instar de l'article de L. Boltanski et P. Bourdieu « Le titre et le poste » (1975) ou, plus récem-

1. L'école à plein temps ou école des métiers est l'équivalent du lycée professionnel français. L'école professionnelle, soit le lieu où se déroule le volet scolaire de la formation en alternance, correspond au CFA. 85,6 \% des CFC sont préparés en apprentissage par alternance (SEFRI, 2014).

2. La centralité du CFC a ici un double sens : celui, objectif, de sa place dans l'histoire des diplômes suisse, mais également dans les représentations mentales de la formation professionnelle helvétique, l'une et l'autre n'étant pas strictement superposables. En ce sens, cet usage de la centralité, qui n'a rien à voir avec une idée de centre ou de moyenne, renvoie à l'usage qui a pu en être fait en sociologie à propos de la centralité du travail dans les sociétés industrielles.

3. http://www.srf.ch/play/tv/eco/video/sergio-ermotti-vor-bewaehrungsprobe?id=39abbae5-9fb4-4d229be9-19268f009acf

4. http://www.vbs.admin.ch/internet/vbs/fr/home/departement/chef.html 
ment, de la polémique entre L'inflation scolaire (Duru-Bellat, 2006) et Le diplôme, arme des faibles (Poullaouec, 2010).

En Suisse, ces perspectives restent également à explorer. Même si les travaux de Philippe Gonon sur la Maturité professionnelle (Kiener et Gonon 1998 ; Gonon, 2013) et l'ouvrage de Muriel Surdez, Diplômes et nation (2005), invitent à stimuler une "sociologie des certifications" (p. 276), les diplômes y sont rarement construits comme objet social à part entière. Cette perspective mérite pourtant d'être retenue à propos du CFC pour interroger sa place, vécue outre-Jura comme centrale : en effet, la Suisse a connu, depuis les années 1990, des réformes de la voie professionnelle qui déstabilisent ce diplôme du fait de l'académisation ${ }^{5}$ des savoirs et de la hiérarchisation accrue des diplômes qui s'observent dans ce pays.

Pour ce faire, il convient de rappeler brièvement l'histoire du CFC, de s'interroger sur la fin du monopole qu'il a longtemps exercé sur la formation professionnelle initiale, en montrant qu'il est désormais un diplôme doublement minoritaire : dans l'ensemble des diplômes mais également dans le sous-ensemble des diplômes professionnels.

\section{Le CFC : un diplôme historique de l'apprentissage}

"Examens et diplômes sont des dispositifs dont les modalités d'action et de distribution ont été forgées progressivement" (Surdez, 2005, p. 268). Ce constat vaut en France (Brucy, 1998) mais également en Suisse, où les premières lois cantonales sur l'apprentissage et l'étatisation des examens sont promulguées entre 1890 et 1906 (Bonoli, 2012 ; Surdez, 2005).

L'USAM (Union suisse des arts et métiers), créée en 1879, jouera un rôle important dans ce processus. Les artisans cherchent en effet, par l'instauration d'un examen de fin d'apprentissage, à résoudre moult contradictions : faire face aux transformations des modes de production, stabiliser les rapports sociaux, se démarquer du travail des usines ou encore préserver un certain élitisme de la formation contre l'interchangeabilité des non-qualifiés. D'abord facultatif, le Certificat de fin d'apprentissage peine à s'imposer : 1501 candidat.e.s en 1877 et 7233 en 1913, soit en moyenne entre 20 et $30 \%$ des jeunes susceptibles d'y concourir (Surdez, 2005, p. 94). Il faut dire que certains cantons, mais également une part importante des artisans eux-mêmes, s'opposent à un processus vu comme contraignant puisque l'inscription à l'examen est liée à la transformation des conditions d'apprentissage (cours complémentaires, durée minimale de formation, etc.).

5. Par académisation, il est entendu, à l'instar de Hanhart (2006) ou Moser (2004), une élévation du niveau d'exigence à l'entrée de la plupart des filières professionnelles ou généralistes. 
En fait, pour s'imposer plus uniformément, la mise en diplômes des savoir-faire exige un prérequis : inscrire le droit de légiférer à propos des métiers artisanaux dans la constitution fédérale, ce qui sera fait en 1908 ; cela suppose aussi des alliés : l'industrie est partagée (Ibidem, p. 124), mais les syndicats de salariés, qui y voient l'occasion de contrer la position "monopolistique " de l'USAM, s'y rallient. Suite aux tensions sociales de la grève générale de $1918^{6}$ et face à la pénurie de main-d'œuvre qualifiée, l'administration fédérale se saisit de la question. Ainsi, à force d'euphémisation des confrontations sociales, un "compromis historique $»^{7}$ (Gonon, 1999, p. 48) débouche sur la loi sur la formation professionnelle de 1930 qui régule nationalement le Certificat fédéral de fin d'apprentissage ${ }^{8}$.

$S$ 'institue ainsi un mode de formation, majoritairement par apprentissage, avec une à deux journée(s) en école professionnelle par semaine, qui, s'il s'est développé initialement dans l'artisanat et le commerce, a peu à peu irradié l'ensemble de l'économie suisse. "L'apprentissage, qui était à l'origine la filière de formation des travailleurs d'élite, est devenu dans le courant des années 50, 60 et 70, la voie normale’ après la scolarité obligatoire" (Gonon, 1999, p. 50). Dans un premier temps, ce diplôme est destiné en priorité aux jeunes hommes suisses (Waardenburg, 2007), mais durant la période des "Trente Glorieuses », il s'adresse non seulement aux femmes, mais également aux jeunes immigré.e.s.

Ainsi, de 18935 CFC distribués en 1936, on passe à 26060 en 1952 et à 30870 en 1960. Pendant cette période, il n'a guère de concurrent au sein du secondaire II. Certes, il existe, depuis les années 1890, la Maturité fédérale', diplôme d'enseignement général conduisant aux universités; mais elle reste réservée à une élite scolaire et sociale, l'orientation vers la voie générale ou professionnelle se faisant, en Suisse, à la fin du primaire, sur la base des résultats scolaires (Cortesi \& Imdorf, 2013, p. 93). Les titulaires de la "Matu " ne sont que 2070 en 1936, 2510 en 1952 et 3320 en $1960^{10}$; elle fait donc peu ombrage à la suprématie du CFC. Destin plus que possible, le CFC s'impose alors comme destin probable (Gonon, 1999), ce qui lui conférera longtemps une position quasi monopolistique. Qu'en est-il aujourd'hui ?

6. En novembre 1918, eut lieu, en Suisse, une grève générale qui mobilisa environ 250000 grévistes et se solda par la mort de trois d'entre eux, tués par l'armée. Après l'arrêt de la grève sur ordre du Conseil fédéral, de nombreuses réformes eurent lieu : réduction massive du temps de travail, mise en œuvre d'un système d'assurances sociales, signature de conventions collectives du travail dans certains secteurs, etc.

7. En lien avec la grève générale, les premières tentatives de paix en matière de travail furent menées à Genève dès 1919. L'enjeu était de créer une situation dans laquelle les conflits collectifs entre employeurs et salariés seraient résolus par la négociation. Cet accord, qui ne se généralise qu’à partir de 1937, devient une caractéristique suisse.

8. La loi de 1930 prévoit déjà, dans son article 40, un " certificat de capacité ». On peut lire l'expression " certificat fédéral de capacité " dans la presse romande dès 1934 (Gazette de Lausanne 22.04.1934), mais l'abréviation CFC apparait dans la presse seulement en 1968 (Gazette de Lausanne 28.6.1968)

9. Equivalent du baccalauréat général, aujourd'hui appelée Maturité gymnasiale.

10. Sources : Office fédéral de la statistique (OFS). 


\section{Encadré 1 : les données mobilisées}

Cette recherche prend appui sur un traitement secondaire des données de l'Office fédéral de la statistique (OFS), qui n'a pas été sans difficultés. En effet, la Suisse est une fédération de 26 cantons ayant leurs propres législations et compétences en matière d'éducation notamment ; elle a donc moins développé de services centralisés de la statistique que la France (Desrosières, 1993).

De plus, l'option prise ici d'une sociologie du diplôme supposait de travailler sur des effectifs de diplômé.e.s (et accessoirement de candidat.e.s) plutôt qu'avec ceux des inscrit.e.s dans telle ou telle formation, statistiques plus fréquentes.

Aussi, et malgré les contacts pris avec l'OFS, il n'est possible de disposer, pour l'ensemble de la Suisse, que de deux types de données sur les diplômes : celles, souvent agrégées et parfois partielles, qui remontent jusque dans les années 1930, et celles, détaillées, couvrant une période allant de 1980 à nos jours. Les premières peuvent fournir des ordres de grandeur. Les secondes permettent des analyses plus précises, mais elles souffrent d'une rupture de nomenclature en 1999.

Cette rupture a conduit à opter pour des principes de catégorisation alignant d'anciens diplômes sur les nouveaux, construisant ainsi des équivalences raisonnées, mais toujours discutables. A titre d'exemples, les Attestations de formation élémentaires ont été assimilées aux Attestations fédérales professionnelles (AFP), créées en 2002, les diplômes des écoles supérieures de commerce (de niveau secondaire II) ont été réunis avec les CFC, tout comme les Baccalauréats internationaux ont été dénombrés avec les Maturités gymnasiales. Le détail des regroupements est précisé lorsque nécessaire. De plus, des diplômes anciens, disparus en 2002, difficiles à situer et peu nombreux ( $n=5286$ en 2003), regroupés dans les statistiques sous l'appellation « diplômes CRS » (Croix-Rouge Suisse), ont été mis hors champ.

\section{Secondaire II : la fin du monopole du CFC}

Sous l'angle des effectifs, le CFC paraît toujours tenir son rang. Sa progression entre 1960 et 1980 est réelle : de 30870 diplômé.e.s en $1960^{11}$, il passe à 53006 en 1980, et affiche une progression toujours effective avec 60016 admis.e.s en 2012. Néanmoins, le détail montre que cet affermissement ne s'est pas fait sans à-coups : les effectifs, après avoir atteint un maximum de 61056 en 1985, chutent à 45815 en 1995, pour ensuite entamer une remontée régulière.

Mais ces résultats globalement favorables camouflent l'essentiel : au cours des trente dernières années, le CFC a perdu le quasi-monopole qu'il exerçait sur le secondaire II (graphique 1) : alors qu'il représentait encore $80 \%$ des diplômes délivrés à ce niveau en 1980 , il ne pèse plus que 60,6 \% en 2012. Le déclin est constant et s'accélère à partir de 1990, année où le CFC regroupe encore 79,2 \% des diplômes de secondaire II.

11. CFC stricto. 


\section{Encadré 2 : quatre catégories de diplômes}

Pour les besoins de l'analyse, quatre grandes catégories de diplômes ont été construites dans l'enseignement supérieur (appelé «tertiaire » en Suisse). Les deux catégories habituellement utilisées (Tertiaire A, pour les Universités, Ecoles polytechniques fédérales et Hautes écoles spécialisées (HES) et Tertiaire B pour la formation professionnelle supérieure, Ecoles techniques supérieures (Technicum)), ont été divisées, afin de mieux faire apparaître les lignes de partage entre formation généraliste et formation professionnelle :

- Le « tertiaire A académique » désigne la voie universitaire classique et regroupe les Bachelor (Licence) et Master, les doctorats et les anciennes Licences et diplômes des Hautes écoles universitaires (HEU), soit 28813 diplômé.e.s en 2012.

- Le « tertiaire A professionnel ou HES » est la nouvelle « figure » de la formation professionnelle de niveau tertiaire ; il est constitué des diplômes des HES (y compris Hautes écoles pédagogiques, HEP) Bachelor (Licence) ou Master, mais également des diplômes post-grades de la formation professionnelle supérieure, soit 19068 diplômé.e.s en 2012.

- Le « tertiaire $B$ » correspond à la formation professionnelle supérieure « historique » et rassemble des diplômes qui relèvent plutôt de la formation continue. Ils ont été conservés dans l'analyse car ils offrent une possibilité de poursuite de formation, voire de promotion, à l'issue d'un CFC. Sont regroupés dans cette catégorie les Brevets, Maîtrises et Diplômes professionnels de niveau tertiaire, à l'exception de ceux en transition vers les HES (en 2001 et avant), et les examens des autres formations professionnelles supérieures. Ils forment un ensemble de 25520 diplômé.e.s (2012).

- Le « tertiaire de spécialisation post académique » est une spécialisation après un cursus académique et réunit les examens professionnels académiques et examens finals universitaires non académiques, ainsi que les diplômes d'études approfondies ou spécialisées universitaires et les diplômes post-grades universitaires d'avant 2005, soit, en 2012 toujours, 2440 diplômé.e.s.

Ce constat renvoie en premier lieu au rapport qu'entretiennent voie professionnelle et voie générale, "distinction essentielle " du système suisse (Cortesi \& Imdorf, 2013, p. 92). En effet, dès les années 1970, "les traits négatifs du 'système dual' [suisse] se dessinèrent [...]. Dans la foulée de l'expansion de l'instruction depuis les années 1960, qui se traduisit avant tout par l'essor des lycées, l'enseignement professionnel fut acculé à justifier sa raison d'être " (Gonon, 1999, p. 50).

De fait, le nombre de Maturités gymnasiales délivrées ne cessera de progresser. Encore rares en 1960 (3 320), elles sont 10370 en 1980 et 19358 en $2012^{12}$. Même si les ordres de grandeur sont difficilement comparables, la différence d'incrémentation dans la société suisse est nette : pour un indice 1 en 1960, le CFC (stricto) passe à 1,9 en 2012, quand les Maturités gymnasiales atteignent 5,8. Le mouvement est inégal : les cantons "latins " et les filles étant plus concerné.e.s par cet essor (Waardenburg, 2011, p. 87). S’il est loin d'inverser l'ordre des choses, ce processus d'académisation du secondaire II est le premier élément qui remet en cause la centralité du CFC.

12. Y compris Baccalauréats internationaux. 


\section{Graphique 1 : le poids du CFC (stricto) dans le secondaire II entre 1980 et 2012 (en \%)}

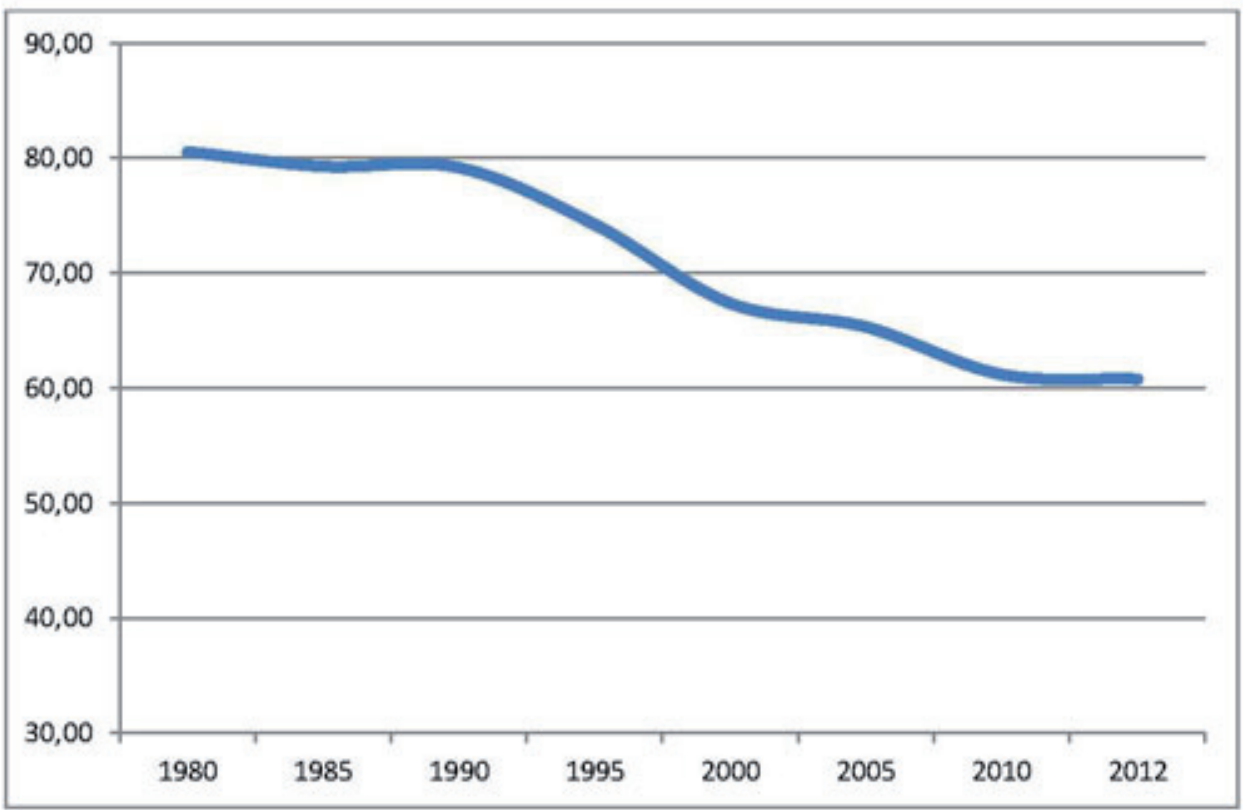

Source : OFS.

Il n'est pas le seul. Au sein même de la formation professionnelle de niveau secondaire II, le $\mathrm{CFC}^{13}$ a cédé du terrain (graphique 2). Entre 1999 et 2012, il perd près de 10 points de pourcentage, en raison du développement de diplômes " concurrents ".

D'un côté, l'Attestation fédérale professionnelle (AFP), mise en place en 2002 en direction des jeunes dont on estime qu'ils ne peuvent pas (ou pas dans l'immédiat) préparer un CFC ; ce diplôme, qui s'obtient en deux ans et qui est supposé permettre de rejoindre ensuite un CFC, prend le relais de l'Attestation de formation élémentaire, qui n'existait qu'au niveau cantonal. Reconnue au niveau fédéral, l'AFP reste d'ampleur modeste, avec environ 5000 diplômé.e.s en 2012 (5 375).

D’un autre côté, le CFC a vu naître, en 1993, un concurrent plus direct, même s'il lui est lié : la Maturité professionnelle. Ce nouveau diplôme, créé de toute pièce, se prépare en complément d'un CFC avec des cours supplémentaires de culture générale ${ }^{14}$ et une durée de 4 ans ou à l'issue d'un CFC en travaillant, en effectuant un stage en entreprise ou dans une école professionnelle à temps-plein pendant un an. Elle se veut un diplôme d'élite

13. Y compris les autres certificats et diplômes des écoles de commerce de niveau secondaire II.

14. Apprentissage de trois langues, mathématiques, et enseignements théoriques spécifiques aux domaines de la Maturité professionnelle. 
avec une grande sélectivité (Waardenburg, 2011, p. 88) et, contrairement au CFC, ouvre directement accès au "Tertiaire A » professionnel (voir Encadré 2). "La Maturité professionnelle est donc un certificat attestant non seulement les compétences professionnelles, mais aussi la capacité à poursuivre des études"(Gonon, 1999, p. 52).

La « menace » est ici plus sérieuse : en 2012, 15696 " Matu pros " ont été délivrées ${ }^{15}$; lors de sa première session, en 1994, il n'y avait eu que 240 lauréat.e.s et 2280 l'année suivante. Nées sous la pression des écoles d'ingénieurs, les Maturités professionnelles ne remettent pas en cause le principe de l'apprentissage, mais se veulent une réponse à un "déficit considérable de connaissances générales et théoriques» (Ibidem, p. 52) reproché au CFC.

Graphique 2 : le poids du CFC dans le secondaire II professionnel entre 1999 et 2012 (en \%)

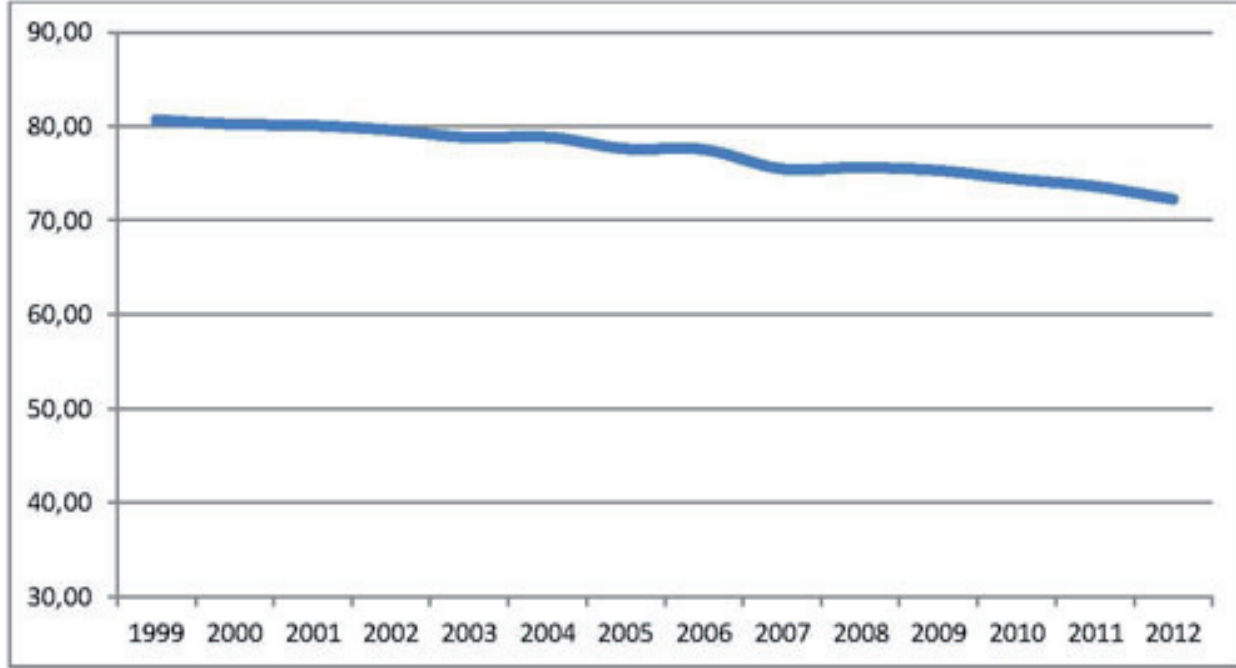

CFC, y compris les autres certificats de capacité et les diplômes des écoles supérieures de commerce de niveau secondaire Il.

Sources: $\mathrm{OFS}$.

Bref, à l'instar de la France avec la création du Baccalauréat professionnel en 1985, la Suisse est convaincue de l'impératif d'élever le niveau de l'enseignement professionnel, dont les manques ont été par ailleurs relevés dans un rapport de l'OCDE (Ibidem, p. 53).

Mais à la différence de l'Hexagone où le "Bac pro " a donné lieu à débats sur la nécessité ou non de lui ouvrir les portes de l'enseignement supérieur (Prost, 2002), la création de la "Matu pro », en Suisse, va de pair avec une réorganisation de l'enseignement

15. Y compris les Maturités spécialisées $(n=2160)$ 
supérieur professionnel, notamment la création des Hautes écoles spécialisées (HES) en 1996. Ces établissements sont pensés comme une destinée possible, voire souhaitable, des nouvelles « Matu pro ».

\section{Encadré 3 : les réformes de la voie professionnelle en Suisse}

1930 : la 1 ère loi permet la constitution d'un cadre de référence national, réunissant les dispositions déjà en vigueur dans certains cantons. Elle est décisive dans l'établissement de formations unifiées dans tout le pays, avec notamment la création du CFC (Certificat fédéral de capacité).

1963 : cette réforme met l'accent sur l'orientation professionnelle des jeunes et le perfectionnement des cadres intermédiaires. La principale nouveauté est la mise en place des Technicum, écoles techniques supérieures (tertiaire B). Pour une formation dans l'ingénierie, deux voies sont dès lors possibles : la voie universitaire (Ecoles polytechniques fédérales) ou la voie professionnelle supérieure (Technicum immédiatement après un CFC).

1978 : les maîtres d'apprentissage sont soumis à une obligation de formation et les associations professionnelles de branche peuvent gérer des cours inter-entreprises permettant aux apprenti-e-s d'avoir des connaissances communes.

Les années 90 : l'ordonnance sur les écoles professionnelles supérieures (1993) et la loi (1995) créant les Hautes écoles spécialisées (HES) témoignent d'une nouvelle filière du supérieur (tertiaire A professionnel, voir Encadré 2). Ces deux textes font référence au nouveau diplôme, qui y donne accès, la Maturité professionnelle, qui fera l'objet d'une ordonnance propre en 1998.

2002 : Toutes les formations non universitaires sont réunies sous la même loi et la voie professionnelle est mieux intégrée dans le système éducatif général. De plus, est introduite, en amont du CFC, une formation initiale en deux ans, sanctionnée par une Attestation fédérale professionnelle (AFP). Enfin, les enseignant.e.s professionnel.le.s sont désormais soumis.e.s à une obligation de formation pédagogique, à l'instar de l'ensemble du corps enseignant.

Le CFC est désormais pris en tenaille : par le bas, avec l'AFP et, par le haut, avec la Maturité professionnelle. Mais ce constat factuel recouvre en fait un double mouvement : d'une part, une élévation du niveau de formation de la jeunesse suisse et, d'autre part, une hiérarchisation et segmentation accrues de la formation professionnelle.

La perte d'influence du CFC dans le secondaire II est corrélée avec une volonté, portée par la réforme de 1993, d'y élever le niveau de formation (Gonon, 1999). La création de la Maturité professionnelle en atteste. La création de l'AFP, quant à elle, fait le pari d'un diplôme préparatoire "tirant " vers le CFC les jeunes qui, sinon, peineraient à y accéder. Mais ces réformes touchent aussi le CFC : ainsi, la loi de 2002 a élevé "le niveau minimum du CFC en supprimant les CFC en 2 ans pour définir la durée de la formation à 3 ou 4 ans " (Waardenburg, 2011, p. 86).

Cette "volonté politique de consolidation du CFC» (Cortesi \& Imdorf, 2013, p. 97) s'est aussi traduite par une extension de son territoire. Ainsi, les diplômes des écoles supérieures de commerce (niveau secondaire II) ont été transformés en CFC et le nombre des 
diplômes non réglementés au niveau fédéral est passé de 7215 à 1387 . Cette élévation du niveau dans le secondaire II a des conséquences au niveau tertiaire, dont l'accès est désormais plus fréquent : entre 1999 et 2012, le nombre de diplômé.e.s du supérieur a presque doublé, passant de 47477 à 80233 ; celui du supérieur professionnel a crû de 30253 à $44588^{16}$. Ce mouvement a été également nourri par la question de l'adhésion à la Communauté Européenne et de "l'euro-compatibilité » éducative de la Suisse (Kiener \& Gonon, 1998), mais également par la publication d'un rapport de l'OCDE montrant que "vue de l'extérieur et hors contexte national, la formation professionnelle [suisse] présente plus de déficits quion ne le pensait jusquiici" (Gonon, 1999, p. 53).

Dès lors, le processus en cours génère une segmentation et une hiérarchisation accentuées de la formation professionnelle suisse. Ce constat est peu souvent discuté. Pourtant, la création, en amont, de l'AFP et en aval, de la "Matu pro ", accroit le processus de sélection au sein du secondaire II professionnel. Tous et toutes ne franchissent pas les paliers que la réforme a mis en place. L'accès au CFC après une AFP n'est pas systématique ${ }^{17}$ et ceux et celles qui sont maintenu.e.s à un niveau infra-CFC risquent une insertion sur le marché de l'emploi précaire ${ }^{18}$; ils rejoindront "la strate 'intermédiaire' sans formation diplômante " (Waardenburg, 2011, p. 95), estimée entre 20 et $25 \%$ d'une génération, selon les études (Amos et al., 2003) ${ }^{19}$.

De même, et malgré la volonté législative de le réformer, le CFC reste un diplôme "hétérogène ", suivant les branches et secteurs d'activité, et dont "les exigences scolaires sont très différentes d'une profession à l'autre " (Ibidem), tant dans les contenus que dans le recrutement ${ }^{20}$. Comme en France (Moreau, 2003), la Suisse connaît une très forte sélection à l'entrée en apprentissage, au regard du sexe (Imdorf, 2004), mais aussi de la nationalité (Fibbi et al., 2003), entretenant ainsi une forte ségrégation horizontale ${ }^{21}$.

16. «Tertiaire professionnel B » (voir Encadré 2) et HES réunis.

17. Selon Infras/Idehap (2010), environ $20 \%$ des titulaires d'une AFP poursuivent en voie CFC et une enquête conduite par le SRED (Bachmann et al., 2014) dans les cantons de Genève et Vaud, montre qu'environ 30 à $40 \%$ des AFP poursuivent en voie CFC.

18. En 2010, le taux de chômage des diplômé-e-s AFP est de 10 \% à 13 \% (Infras/Idehap, 2010), alors que la moyenne Suisse des 20-25 ans se situe à environ 5,5 \% (OFS/SECO, 2011).

19. Les statistiques de l'OFS estiment le nombre de jeunes " ayant terminé leur scolarité prématurément » à entre 6 et $11 \%$ suivant les années (période 1990-2011).

20. En Suisse, les branches professionnelles jouent un rôle primordial dans la définition des contenus de formation et référentiels de diplômes ; de même, leurs latitudes sont grandes dans le choix des apprentis qu'elles recrutent.

21. Par ségrégation horizontale, il est entendu l'inégale répartition des femmes et des hommes dans les différents métiers ou filières de formation y conduisant. On assiste en effet à une concentration des femmes dans quelques secteurs et surtout une quasi non-mixité dans la plupart des domaines d'activité. En outre, les secteurs dans lesquels se dirigent les femmes sont moins rémunérés, moins reconnus socialement et offrent moins de perspectives de carrière. 


\section{Des appropriations différenciées des réformes du secondaire II}

Lors de la création de l'AFP (2002), aucune obligation ne contraignait les associations professionnelles de branches à proposer un tel diplôme. Ainsi, les arts appliqués, les arts et la musique ne proposent pas de formation AFP. Les secteurs de l'alimentation, de l'artisanat, du commerce et de la vente, de la construction et des transports y recourent plus massivement. Entre les deux, les secteurs de l'industrie, les métiers verts, la santé et le social n'ont que peu saisi cette possibilité2 ${ }^{22}$

Les arts appliqués, mais surtout les arts et la musique, ont connu un processus de professionnalisation très récent (création du CFC de danseur ou de comédien) et ont probablement décidé de se concentrer uniquement sur la mise en place de ce diplôme, nouveau pour eux.

A contrario, les secteurs traditionnellement engagés dans l'apprentissage et le CFC (bâtiment, artisanat, alimentation) ont très fortement adhéré à l'AFP. Mais d'autres raisons expliquent cet engagement. En effet, si les métiers de l'alimentation ou du bâtiment sont de grands pourvoyeurs de places d'apprentissage, ce sont également des secteurs délaissés par les jeunes où des places restent vacantes (LINK, 2013), et qui connaissent des taux de rupture d'apprentissage très élevés (restauration : 35-40\% ; bâtiment : 25-30\% ; voir à ce propos Badel, 2013 ; Lamamra et Masdonati, 2009 ; Rastoldo et al., 2009 ; Stalder et Schmid, 2006). Ces secteurs se distinguent aussi par le recours à une proportion élevée de main-d'œuvre non qualifiée. La création d'un diplôme inférieur au CFC peut ainsi traduire une volonté de réguler le marché du travail en termes de recrutement et de formation.

Les secteurs qui proposent peu d'AFP, comme l'industrie, répondent probablement à des logiques inverses. Celle-ci a, en effet, connu une élévation de son niveau de qualification, liée à l'informatisation et la technologisation des procès de production. Preuve en est le nombre important de Maturités professionnelles industrielles. Le niveau d'exigence élevé rend dès lors difficile de proposer des voies AFP.

Enfin, pour les professions de la santé et du social, arrivées récemment dans le système de formation professionnelle, la création de certains CFC a déjà suscité de fortes résistances. Dans un domaine déjà fragmenté et hiérarchisé, les nouveaux métiers associés au CFC doivent coexister avec, et s'intercaler entre, des métiers issus des anciens systèmes de formation (écoles supérieures, formations Croix-Rouge, etc.).

22. Pour identifier les secteurs ayant mis en place ou non une filière AFP, $c f$. les listes des professions éditées par le Secrétariat d'Etat à la formation, à la recherche et à l'innovation (SEFRI), voir : http://www.sbfi. admin.ch/bvz/grundbildung/index.html?lang=fr. 
Dès lors, la mise en place des AFP dans certaines branches a surtout révélé une logique de spécialisation, une segmentation et une hiérarchisation accrues. Les nomenclatures utilisées pour ces nouvelles filières le montrent bien : " aide ", "assistant.e ", "employé.e ", ou " praticien.ne ", sont des termes qui soulignent le caractère subalterne dans la division du travail assigné aux futur.e.s titulaires de ce diplôme.

La création de la Maturité professionnelle répond à des enjeux différents : offrir des perspectives de formation post-CFC pour les apprenti.e.s qui souhaiteraient poursuivre vers le tertiaire, mais aussi proposer un espace de formation aux futurs cadres de l'industrie (Kiener \& Gonon, 1998).

Si dans un premier temps, le développement des écoles professionnelles supérieures est lié à l'essor des Technicum (voir encadré 3), et donc indirectement à l'industrie, la mise sur pied d'une formation unifiée au niveau fédéral, puis d'un diplôme commun, va étendre la formation professionnelle supérieure à d'autres branches, notamment les emplois de service.

Ainsi, dans la loi, la Maturité professionnelle est ouverte à toute personne débutant ou ayant achevé une formation sanctionnée par un CFC. Cela laisse supposer que la formation professionnelle initiale dans n'importe quel métier permet d'obtenir ce diplôme.

Pourtant, deux types de "Matu pro », la commerciale (52\%) et la technique (34\%), se taillent la part du lion (OFS, 2007). De fait, il existe un lien entre formation initiale suivie (ou métier appris) et probabilité de poursuivre vers une "Matu Pro " et donc vers le tertiaire A. L'accès à la Maturité professionnelle dépend "fondamentalement, $d u$ choix de la profession". "Alors que $60 \%$ des électroniciens et $58 \%$ des diplômés d'écoles supérieures de commerce obtiennent une maturité professionnelle, c'est le cas de seulement $5 \%$ des mécaniciens d'automobiles et de $2 \%$ des vendeurs. " (Cortesi \& Imdorf, 2013, p. 102). En 2010, ces tendances se confirment (OFS, 2011) : tous les CFC ne sont pas égaux face aux enjeux d'élévation et d'académisation.

Si la création d'une Maturité professionnelle permet de poursuivre sa formation au niveau tertiaire, et ce, pas uniquement dans le tertiaire $\mathrm{B}$, elle dessine de facto une ligne de faille au sein des CFC. Derrière un diplôme jusqu'ici présenté comme unifié apparaissent des différences d'avenir.

L'introduction de la Maturité professionnelle a donc doublement déstabilisé la position hégémonique du CFC, d'une part, en lui conférant, en partie du moins, un statut de diplôme propédeutique et, d'autre part, en soulignant son hétérogénéité. En cela, le maintien des effectifs en CFC est illusoire. En perdant sa position hégémonique au sein du secondaire II, il pâtit d'une volonté politique d'élévation du niveau de formation, observée dans la plupart des pays occidentaux et à laquelle la Suisse ne fait pas exception. 


\section{Le CFC, un diplôme désormais minoritaire}

Sur trente ans, et rapporté à l'ensemble des diplômes distribués, le déclin du CFC est impressionnant (graphique 3). Alors qu'il représentait les deux tiers des diplômé.e.s en 1980 (66,5\%), il n'en regroupe plus qu'un tiers en 2012 (35,8\%).

C'est à la fin des années 1980 que débute ce processus, particulièrement marqué entre 1990 et 2000 : le CFC représentait encore 61,1 \% des diplômes distribués en 1990 mais chute à $47,8 \%$ en 2000. Et si depuis, la baisse est en peu enrayée, la rupture est effective : le CFC n'est plus ce qu'il était.

Or, l'essor de la Maturité gymnasiale et consécutivement celui de la voie universitaire académique, n'expliquent pas, à eux seuls, cet " effacement " du CFC dans le paysage des diplômes suisses.

Son déclin doit également à la tertiarisation de la formation professionnelle via l'essor des Hautes écoles spécialisées et des diplômes du supérieur associés. Alors que le CFC représentait $85,2 \%$ des diplômes distribués dans la voie professionnelle (secondaire et tertiaire réunis) en 1980, il n'en regroupe plus que 49,5\% en 2012 (graphique 4). A nouveau, la barre symbolique de la majorité est franchie, en négatif. S'il reste puissant, le CFC n'est plus, dans ses terres de prédilection - la voie professionnelle -, le diplôme magistral d'il y a trente ans.

Le tertiaire professionnel s'en trouve affecté. $\mathrm{Si}$, comme indiqué supra, il bénéficie d'un net accroissement du nombre de ses diplômé.e.s, le profit est surtout marqué pour le «tertiaire A professionnel " plutôt que pour le «tertiaire $\mathrm{B}$ » $^{23}$, puisque le premier passe, de 1999 à 2012, de 7366 lauréat.e.s à 19 068, quand le second stagne (respectivement 22887 et 25520 ).

Le risque de disjonction au sein de la voie professionnelle de niveau supérieur entre les Brevets et Diplômes professionnels, marqués historiquement du sceau de la promotion sociale, et les Hautes écoles spécialisées, plus pénétrées du modèle académique et où dominent les Bachelor (Licence) et les Master, est réel et ajoute à l'éclatement de la voie professionnelle en créant une "barrière " au sein du tertiaire professionnel. Derrière la fin de la centralité du CFC, c'est tout un échafaudage qui se déséquilibre.

23. Cf. Encadré 2. 
Graphique 3 : le poids du CFC (stricto) dans l'ensemble des diplômes du secondaire et du tertiaire (supérieur) entre 1980 et 2012 (en \%)

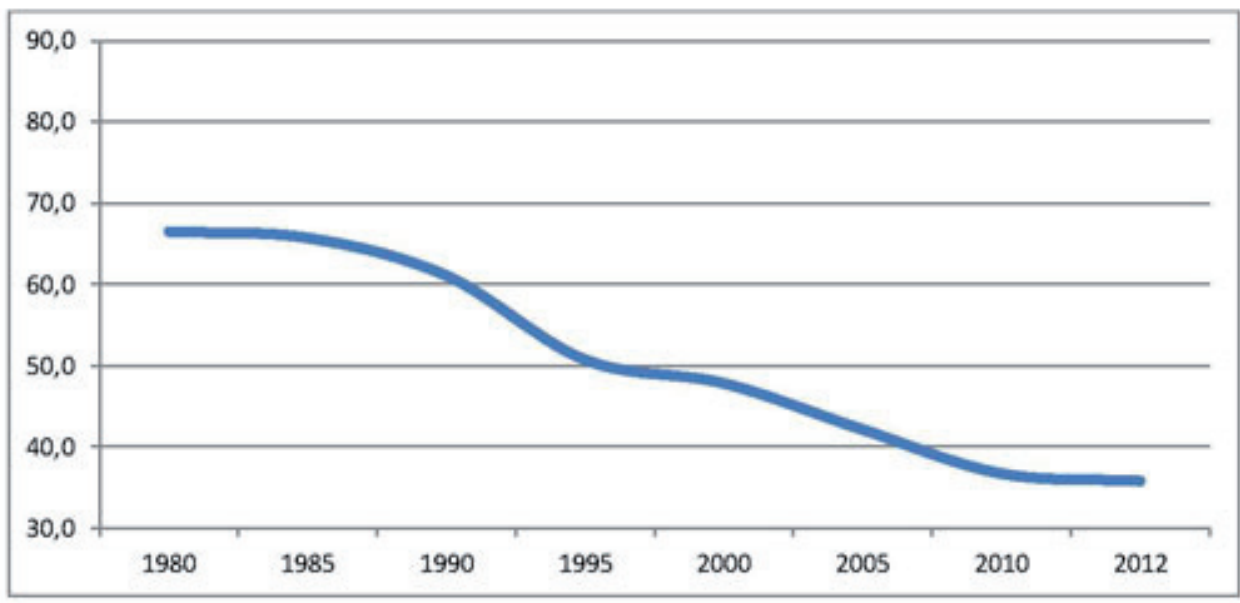

Sources: OFS

Graphique 4 : le poids du CFC (stricto) dans l'ensemble des diplômes professionnels ${ }^{24}$ entre 1980 et 2012 (en \%)

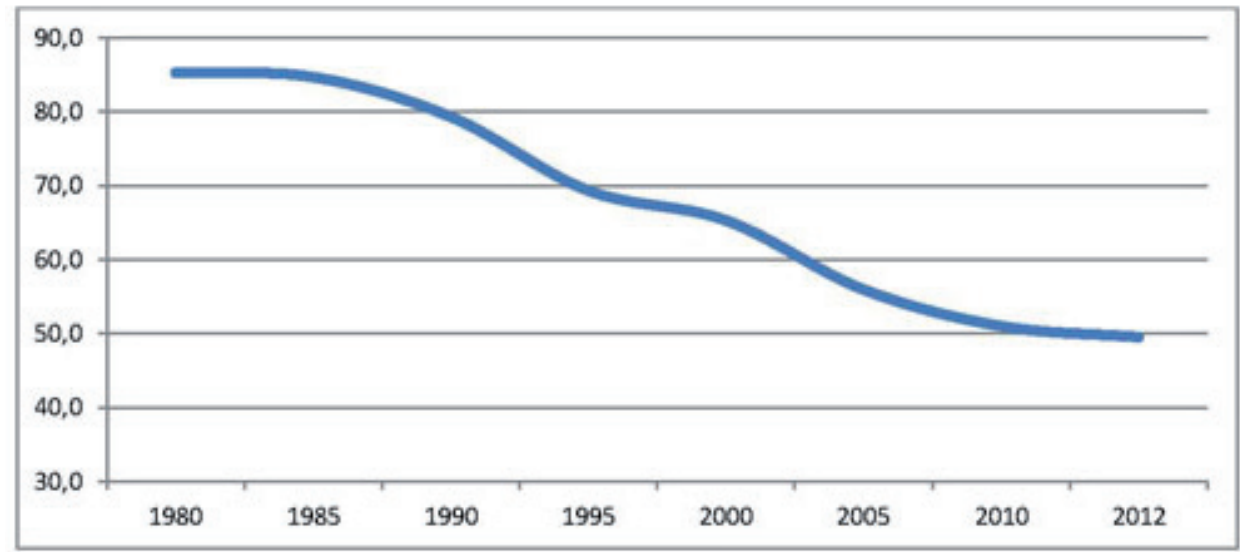

Source : OFS.

24. Soit les diplômes professionnels du secondaire II, du tertiaire A professionnel et du tertiaire B (Cf. Encadré 2). 
Désormais doublement minoritaire, dans l'ensemble des diplômes et dans celui des diplômes professionnels, le CFC n'est plus, objectivement, au cour de la formation suisse. Comme d'autres pays, la Suisse est devenue une "société des diplômes " (Millet, Moreau, 2011), et sur cet échiquier nouveau, le CFC fait moins figure de pièce centrale.

L'accroissement du nombre de diplômes distribués chaque année y contribue (graphique 5) : depuis le début du XXI ${ }^{\mathrm{e}}$ siècle, il est passé de 128860 (en 2000) à 188139 (en 2012). En 1980, il avoisinait les $80000^{25}$. En œuvre avant même les réformes des années 1990, bien qu'amplifiées par elles, l'élévation, l'académisation et la multiplication des diplômes suisses ont été préparées de longue date.

Ce mouvement n'est pas imputable à des effets démographiques ${ }^{26}$. Il a en revanche un effet sociodémographique sans doute imprévu : les femmes bénéficient plus, ou sont plus actrices, de cette diplômation de la société suisse.

En effet, depuis le début du XXI ${ }^{\mathrm{e}}$ siècle, l'accroissement du nombre de diplômé.e.s leur a permis de réduire sensiblement l'écart avec les hommes (graphique 5) : en 1999, l'amplitude entre diplômés et diplômées était de 17 031, au bénéfice des premiers ; en 2013, elle n'est plus que de 4051.

La généralisation des diplômes contribuerait-elle à l'égalité des sexes ? Partiellement, tant elle peine, dans un pays où la ségrégation horizontale est particulièrement marquée (OFS, 2008), à remettre en cause les assignations sexuées des métiers, des formations (Lamamra, 2011) et donc le sexe des diplômes. Ainsi, dans le secondaire II, l'AFP et le CFC restent marqués du sceau du masculin avec des taux de féminisation respectifs de 40,6 \% et 45,9\% (2012), quand la Maturité gymnasiale (56,2\%) et les Maturités spécialisées $^{27}(82,4 \%)$ développent un tropisme féminin. Dans le tertiaire, les Licences et diplômes des Hautes écoles universitaires $(65 \%)$ et, dans une moindre mesure, les Bachelor et Master (53,1 \% et 52,2\%), sont les formations les plus féminisées ${ }^{28}$, quand les Brevets et Diplômes professionnels de la filière « tertiaire professionnel B » conservent une tonalité plus masculine (41,9\% de femmes en 2012).

25. 81315 ou 79715 suivant les sources OFS.

26. Selon le recensement fédéral (OFS, 2015), la population résidante était de 6335243 habitant.e.s en 1980, de 7204055 en 2000 et de 8139631 en 2013. Cela signifie, pour une base 100, une augmentation de 13 points de pourcentage entre 2000 et 2013, pendant que l'augmentation du nombre de diplômes (voir chiffres supra) durant ce même laps de temps est de 46 points. Si on étend la période de 1980 à 2013, l'augmentation de la population est de 28 points, celle des diplômes de 135 points.

27. Sous-ensemble des Maturités professionnelles destinées aux personnes ayant suivi une école de culture générale ou une école de commerce et préparant l'accès aux HES. Elles sont spécialisées, selon les cantons, dans les arts visuels, la communication et l'information, la musique, la pédagogie, le social ou la santé.

28. A l'exception du doctorat où la proportion de femmes est de 43,2 \%. 
Graphique 5 : le nombre de diplômes distribués en Suisse entre 1999 et 2012

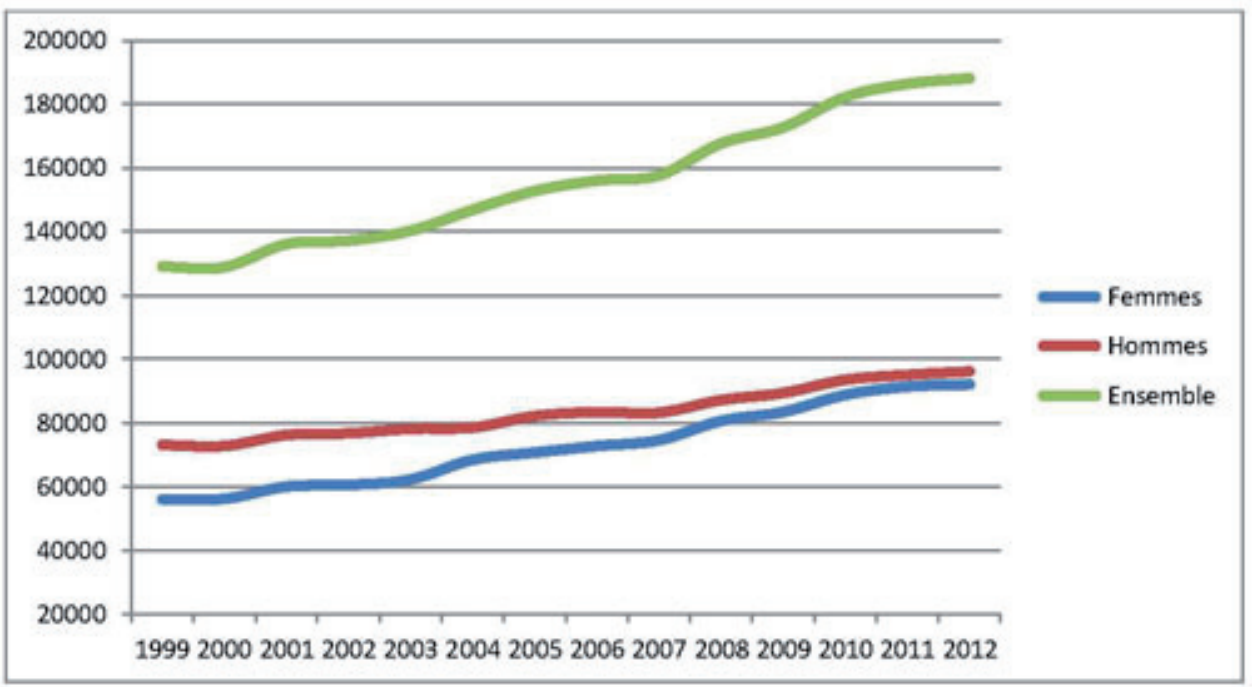

Sources: OFS.

\section{Conclusion}

Dans une société suisse qui ne cesse d'accroître le nombre de diplômes distribués chaque année, et qui le fait par la diversification du secondaire II et par l'essor du niveau tertiaire, la place du CFC a indéniablement été remaniée, même si l'illusion de sa centralité est soigneusement entretenue.

Valorisé en interne, mais également à l'étranger (Garçon, 2014), le diplôme phare de la formation professionnelle semble toujours au cœur du système suisse. Pourtant, s'il reste à des niveaux d'effectifs élevés, le CFC n'a plus la centralité qui fut longtemps la sienne dans l'espace des diplômes suisses. Concurrencé en amont et en aval au sein du secondaire II, bousculé par la tertiarisation de la voie professionnelle, il n'est désormais plus majoritaire, dans l'ensemble des diplômes distribués, mais également au sein du sous-ensemble des diplômes professionnels. Il tend également à devenir propédeutique quand, longtemps, il a été avant tout un diplôme d'insertion.

Une conclusion logique s'impose donc : le déclin (proportionnel) du CFC et la stagnation $\mathrm{du}$ " tertiaire B » (formation professionnelle supérieure traditionnelle, écoles techniques supérieures (Technicum)), confrontés à la montée en puissance du " tertiaire $A$ » (Universités, Ecoles polytechniques fédérales et Hautes écoles spécialisées (HES)), risquent de conduire à une baisse du nombre de jeunes qui passent par l'apprentissage pour se former. Bref, la " diplômation " de la Suisse va de pair avec une " scolarisation " de ses formations et donc ... avec la fin de la centralité de l'apprentissage. L'hypothèse n'est pas 
nouvelle : Philippe Gonon la formulait dès 1999 : "à long terme, l'importance du modèle de formation duale ira en diminuant" (p. 53). Quinze ans après, elle semble se vérifier.

Dans cette «société des diplômes " qu'est devenue la Suisse, le CFC est un diplôme parmi d'autres, lesquels autres offrent souvent de meilleures perspectives, en termes de position occupée dans la division du travail. Par ailleurs, son statut de diplôme d'insertion est remis en cause par la création de la Maturité professionnelle, dont il est devenu la propédeutique, instituant ainsi le CFC en diplôme à double finalité. Ainsi dédoublé et désormais minoritaire, il risque au final de perdre la " communauté de confiance " (Caillaud, 2013) qu'il avait, historiquement, cristallisée sur son nom.

\section{Bibliographie}

Amos J., Böni E., Donati, M., Hupka S, Meyer, T. \& Stalder B. E. (2003), Parcours vers les formations postobligatoires. Les deux premières années après l'école obligatoire. Résultats intermédiaires de l'étude longitudinale TREE, OFS, Neuchâtel.

Bachmann Hunziker K., Leuenberger Zanetta S., Mouad R., Rastoldo F. \& Charmillot G. (2014), Que font les jeunes 18 mois après l'obtention de leur diplôme de niveau secondaire II ? Etat des lieux dans les cantons de Vaud et de Genève, SRED, Genève.

Badel P.-H. (2013), "Ruptures de contrats, quelles causes et quels remèdes ? "Context, $\mathrm{n}^{\circ}$ 6-7, pp. 6-7.

Boltanski L. \& Bourdieu P. (1975), « Le titre et le poste. Rapports entre le système de production et le système de reproduction ", Actes de la recherche en sciences sociales, vol. 1, $\mathrm{n}^{\circ} 1.2$, pp. 95-107.

Bonoli L. (2012), « La naissance de la formation professionnelle en Suisse : entre compétences techniques et éducation morale ", Education Permanente, n 192(3), pp. 209-221.

Brucy G. (1998), Histoire des diplômes de l'enseignement technique et professionnel (18801965), Belin, Paris.

Brucy G., Maillard F. \& Moreau G. (dir.) (2013), Le CAP. Un diplôme du peuple (19112011), Presses universitaires de Rennes, Rennes.

Cabannel P. (2002), La République du certificat d'études. Histoire et anthropologie d'un examen (XIX-XXe siècles), Belin, Paris.

Caillaud P. (2013), "L'Europe des certifications professionnelles : coordination des systèmes nationaux ou promotion d'un modèle européen ? ", Cahiers de la recherche sur l'éducation et les savoirs. Les 'petits' diplômes professionnels en France et en Europe, Horssérie 4, pp. 33-50. 
Cortesi S. \& Imdorf C. (2013), "Le certificat fédéral de capacité en Suisse - Quelles significations sociales pour un diplôme hétérogène ? ", Cahiers de la recherche sur l'éducation et les savoirs. Les 'petits' diplômes professionnels en France et en Europe, Hors-série 4, pp. 91-108.

Desrosières A. (1993), La politique des grands nombres. Histoire de la raison statistique, La Découverte, Paris.

Duru-Bellat M. (2006), L'inflation scolaire, Seuil, Paris.

Fibbi R., Kaya B. \& Piguet E. (2003), Nomen est omen: Quand sappeler Pierre, Afrim ou Mehmet fait la différence. Direction du programme PNR 43 en collaboration avec le Forum Formation et emploi et le Centre suisse de coordination pour la recherche en éducation (CSRE), Bern/Aarau.

Garçon F. (2014), Formation : l'autre miracle Suisse. Universités - Ecoles polytechniques HES - Apprentissage, Presses polytechniques et universitaires romandes, Lausanne.

Goblot E. (1925), La barrière et le niveau. Etude sociologique sur la bourgeoisie française moderne, Presses universitaires de France, Paris.

Gonon Ph. (2013), "Federal Vocational Baccalaureate: The Swiss Way of Hybridity", In: Deissinger Th., Aff J., Fuller A. \& Helms Jorgensen Ch. (Eds.), Hybrid Qualifications - Structures and Problems in the Context of European VET Policy. Peter Lang, Berne, pp. 181-196.

Gonon Ph. (1999), "Les efforts actuels de réforme de l'enseignement professionnel en Suisse ", Formation professionnelle, n 17 , pp. 48-54.

Hanhart S. (2006), " Marché de l'apprentissage et pouvoirs publics », Bulletin de la CIIP, $\mathrm{n}^{\circ} 19$, pp. 8-9.

Infras/Idheap (2010), Evaluation de la formation professionnelle initiale de deux ans (AFP).

Rapport final et annexe réalisés sur mandat de l'Office fédéral de la formation professionnelle et de la technologie, OFFT, Berne.

Imdorf C. (2004), «La sélection fondée sur le genre dans l'attribution des places de formation ", in CSDE (Ed.), Le genre en vue. Les filles et les jeunes fermmes face à la formation, Conférence suisse des déléguées à l'égalité, Berne, pp. 107-120.

Kaplan S. (2001), La fin des corporations, Fayard, Paris.

Kiener U. \& Gonon Ph. (1998), La maturité professionnelle comme illustration de la politique suisse en matière de formation professionnelle. Rapport de valorisation, Direction du Programme national de recherche 33 (PNR 33) et Centre Suisse de coordination pour la recherché en éducation (CSRE), Berne/Aarau. 
Lamamra N \& Masdonati J. (2009), Arrêter une formation professionnelle: Mots et maux d'apprenti-e-s, Antipodes, Lausanne.

Lamamra N. (2011), " La formation professionnelle en alternance, un lieu de transmission des normes de genre ? ", Revue Psychologie du travail et des organisations, ${ }^{\circ} 17(4)$, pp. 330-345.

LINK (2013), Baromètre des places d'apprentissage, Secrétariat d'Etat à la formation et à l'innovation - SEFRI, Berne.

Maillard F. (dir.) (2008), Des diplômes aux certifications professionnelles, Presses universitaires de Rennes, Rennes.

Marchand P. (dir.) (2010), "Le baccalauréat, 1808-2008. Certification française ou pratique européenne?", Hors série Revue du Nord, collection " Histoire ", n 24.

Millet M. \& Moreau G. (Dir.), La société des diplômes, La Dispute, Paris.

Moreau G. (2003), Le monde apprenti, La Dispute, Paris.

Moser U. (2004), Jugentliche zwischen Schule und Berufsbildung: Eine Evaluation bei Schweizer Grossunternehmen unter Berücksichtigung des internationalen Schulleistungsvergleichs PISA Leitungsgruppe des NFP 43, Retrieved June 01, 2005, from http://www.nfp43.unibe.ch

Müller B. \& Schweri J. (2006), Die Entwicklung der betrieblichen Ausbildungsbereitschaft. Eine Längsshnittuntersuchung zur dualen Berufsbildung in der Schweiz. (SIBP Schriftenreihe Nr. 31), Schweizerische Institut für BerufsPädagogik, Zollikofen.

Office fédéral de la statistique - OFS (2007), Maturités et passages vers les hautes écoles. Neuchâtel : OFS.

Office fédéral de la statistique - OFS (2008), Vers l'égalité 2008. Women and men in OECD countries, Retrieved 03.08.2010, from http://www.oecd.org/dataoecd/44/52/37962502. pdf

Office fédéral de la statistique - OFS / Secrétariat d'Etat à l'économie - SECO (2011), Le chômage en Suisse 2011. Demandeurs d'emploi et chômeurs inscrits. Bénéficiaires de prestations de l'assurance-chômage, OFS, Neuchâtel.

Office fédéral de la statistique - OFS (2015), Relevé structurel du recensement fédéral de la population 2013, OFS, Neuchâtel.

Poullaouec T. (2010), Le diplôme, arme des faibles. Les familles ouvrières et l'école, La Dispute, Paris.

Prost A. (2002), "La création du baccalauréat professionnel : histoire d'une décision ", in Moreau G. (dir.), Les patrons, l'Etat et la formation des jeunes, La Dispute, Paris, pp. 95-111. 
Rastoldo F., Amos J. \& Davaud C. (2009), Les jeunes en formation professionnelle. Rapport III : Le devenir des jeunes abandonnant leur apprentissage, SRED, Genève.

Stalder B. E. \& Schmid E. (2006), Lehrvertragsaufösungen, ihre Ursachen und Konsequenzen: Ergebnisse aus dem Projekt LEVA Bildungplanung und Evaluation, Bildungplanung und Evaluation der Erziehungsdirektion des Kantons Bern, Bern.

Surdez M. (2005), Diplômes et nation. La constitution d'un espace suisse des professions avocate et artisanales (1880-1930), Peter Lang, Berne.

Waardenburg G. (2007), « Passer de l'école au travail productif. La reproduction sociale par la voie suisse de l'apprentissage professionnel », in Roca i Escoda M. \& Panayotopoulos N. (Eds.), Structures sociales en transformation. Approches théoriques, méthodologiques et empiriques. Actes de la II' Ecole d'été ESSE, université de Crète, 102-111.

Waardenburg G. (2011), "Le système suisse de diplôme ", in Millet M. \& Moreau G. (Eds.), La société des diplômes, La Dispute, Paris, pp. 81-96. 